\title{
Two-dimensional general fan-type FIR digital filter design
}

\author{
Soo-Chang Pei*, Sy-Been Jaw \\ Department of Electrical Engineering, National Taiwan University, Taipei, Taiwan, ROC
}

Received 13 May 1991; revised 2 March 1992, 22 July 1993 and 25 October 1993

\begin{abstract}
Conventional transform methods can only design ordinary and quadrant fan-type filters with $90^{\circ}$ pass-angle. In this paper, three efficient approaches are proposed for designing general arbitrary pass-angle fan-type filters. They include the analytic closed form, the rotated, and the frequency sampling design methods. Each approach has its features and is very suitable for directional filtering and image processing applications.

\section{Zusammenfassung}

Konventionelle Transformationsmethoden ermöglichen nur den Entwurf von gewöhnlichen und Quadrant Fan-Typ Filtern mit $90^{\circ}$ Durchlaßphase. In dieser Arbeit werden drei effiziente Lösungen für den Entwurf von allgemeinen Fan-Typ-Filtern mit beliebigen Durchlaßwinkeln vorgeschlagen. Sie umfassen die analytische geschlossene Form, die Rotations- und die Frequency Sampling Entwurfsmethode. Jeder Entwurf hat seine Vorteile und ist sehr gut zur richtungsabhängigen Filterung und für Bildverarbeitungsanwendungen geeignet.

\section{Résumé}

Les méthodes de transformation conventionnelle ne permettent que la description des filtres quadrants et ordinaire d'angle de passage à $90^{\circ}$. Dans cet article, trois approches efficaces sont proposées pour définir des filtres généraux à angle de passage arbitraire. Ces approches utilisent les méthodes basées sur lar forme analytique close, la rotation, et la description d'échantillon fréquentielle. Chaque approche possède ses propres caractéristiques et est très bien adaptée au filtrage directionnel et aux applications de traitement d'images.
\end{abstract}

Key words: FIR filter; Fan-type filter

\section{Introduction}

Fan-type filter is a special two-dimensional (2D) digital filter. It has the capability of directional filtering. A signal is passed or rejected according to

* Corresponding author. its direction. Direction is a special characteristic of 2D signal, it cannot be defined for 1D signal. Fan-type filters have been used in many applications such as geological and seismological data processing.

There are three major kinds of conventional fantype filters: one is the ordinary fan-type filter (Fig. 1(a)), another is the quadrant fan-type filter 

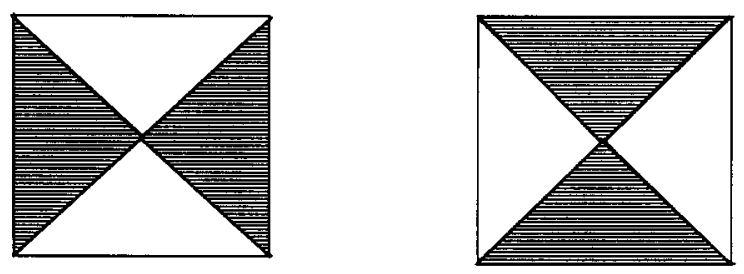

(a) Ordinary fan-type filters.
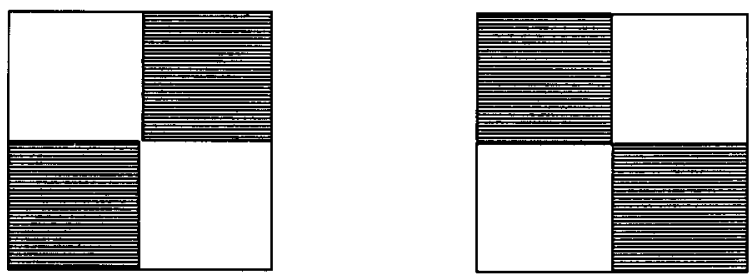

(b) Quadrant fan-type filters.

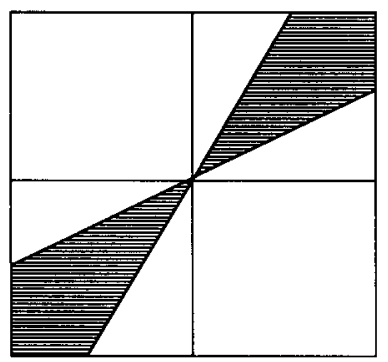

(c) General fan-type filter.

Fig. 1. Three major kinds of conventional fan-type filters. (a) Ordinary fan-type filters. (b) Quadrant fan-type filters. (c) General fan-type filter.

(Fig. 1(b)), the other is the general fan-type filter (Fig. 1(c)). The first two types are also called $90^{\circ}$ fan filters. So far, several conventional methods have been developed to design $90^{\circ}$ fan filters. The most popular method is the McClellan transformation $[15,16]$, it uses a simple formula to map a $1 \mathrm{D}$ filter into a $2 \mathrm{D}$ filter which still preserves the characteristic of the 1D filter. Another method is the complex transformation which is based on spectrum translations and rotations $[2,4,9,10,17]$. Generally, complex transformation is more complicated than the McClellan transformation, but it can be applied for both IIR and FIR fan filters. The advantage of the McClellan and complex transform methods is that it reduces a 2D design problem to a $1 \mathrm{D}$ design which is much easier to handle. However, these two methods can design only ordinary and quadrant fan-type filters, a simple network transformation has been proposed to design general fan-type filters [7]. But this method has some non-linear bending effects in high frequency components due to bilinear transform [13]. Another approach $[5,14]$ is to use the $L_{p}$ optimization technique to design general FIR and IIR fan-type filters with arbitrary pass-angle. This optimization approach is more general than the transform methods, but it is complicated and time consuming due to the optimization procedures involved.

In this paper, three efficient approaches are proposed for designing arbitrary pass-angle fan-type filters. They are much simpler and more effective than the optimization method; also there are the analytic closed form solutions in the two proposed approaches. These fan-type filters are very useful for directional filtering and image coding applications.

\section{Analytic closed form fan-type filter}

\subsection{Definition of general fan-type filter}

Fig. 2 illustrates the specifications for the general fan-type filter design, the shaded region is the passband and the rest of the unshaded region is the stopband. The passband is bounded by two straight boundary lines which pass through the origin; these two boundary lines can point to arbitrary directions. The parameter $B$ is the passband width which is greater than 0 and less than or equal to $\pi$. The pass angle $\theta$ is the spread angle of the passband. In ordinary and quadrant fan-type filters, the pass angle is fixed to be $90^{\circ}$, but it can be arbitrarily assigned from $0^{\circ}$ to $180^{\circ}$ for general fan-type filters. The passband width $B$ is used to control the aliasing effect which is the leakage beyond the folding frequency and reflects back into the other end.

\subsection{Ideal impulse response of general fan-type filter}

In order to calculate the ideal impulse response of a general fan-type filter [6], it is convenient to 


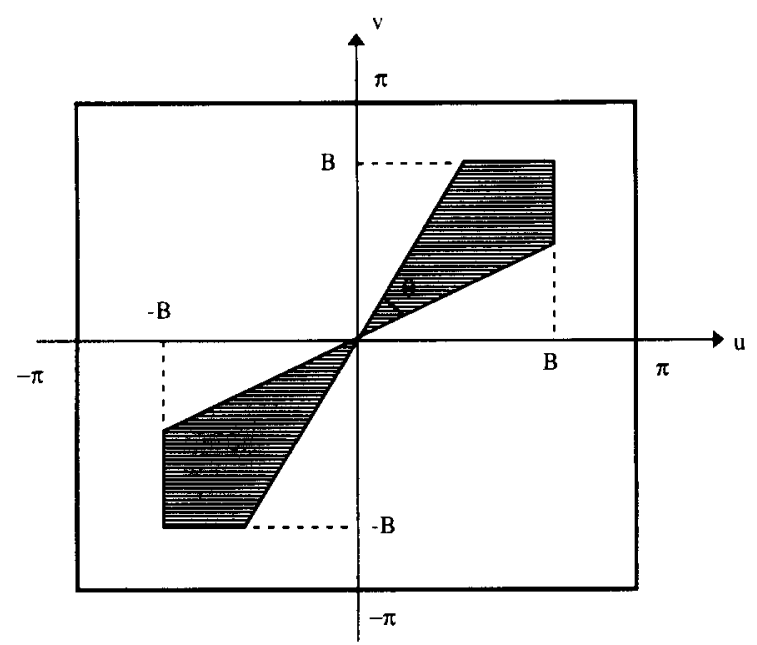

Fig. 2. Specification of general fan-type filter.

divide frequency domain into two regions $R_{1}$ and $R_{2}$ as shown in Fig. 3. No matter which region the passband locates in, the passband can always be represented by a shaded triangle in each region, as depicted in Fig. 4. Assuming

$H\left(\mathrm{e}^{\mathrm{j} u}, \mathrm{e}^{\mathrm{j} v}\right)=1$ for passband,

$H\left(\mathrm{e}^{\mathrm{j} u}, \mathrm{e}^{\mathrm{j} v}\right)=0$ for stopband,

then the ideal impulse response $h(m, n)$ can be written as

$$
\begin{aligned}
h(m, n)= & \frac{1}{4 \pi^{2}} \int_{0}^{\pi} \int_{-u}^{u}\left[H\left(\mathrm{e}^{\mathrm{j} u}, \mathrm{e}^{\mathrm{j} v}\right) \mathrm{e}^{\mathrm{j}(m u+n v)}\right. \\
& \left.+H\left(\mathrm{e}^{-\mathrm{j} u}, \mathrm{e}^{-\mathrm{j} v}\right) \mathrm{e}^{-\mathrm{j}(m u+n v)}\right] \mathrm{d} u \mathrm{~d} v \\
& +\frac{1}{4 \pi^{2}} \int_{0}^{\pi} \int_{-v}^{v}\left[H\left(\mathrm{e}^{\mathrm{j} u}, \mathrm{e}^{\mathrm{j} v}\right) \mathrm{e}^{\mathrm{j}(m u+n v)}\right. \\
& \left.+H\left(\mathrm{e}^{-\mathrm{j} u}, \mathrm{e}^{-\mathrm{j} v}\right) \mathrm{e}^{-\mathrm{j}(m u+n v)}\right] \mathrm{d} u \mathrm{~d} v \\
= & \frac{1}{4 \pi^{2}} \int_{0}^{B} \int_{a u}^{b u} 2 \cos (m u+n v) \mathrm{d} u \mathrm{~d} v \\
& +\frac{1}{4 \pi^{2}} \int_{0}^{B} \int_{c v}^{d v} 2 \cos (m u+n v) \mathrm{d} u \mathrm{~d} v
\end{aligned}
$$

The four parameters $a, b, c, d$ are defined in Fig. 4 .

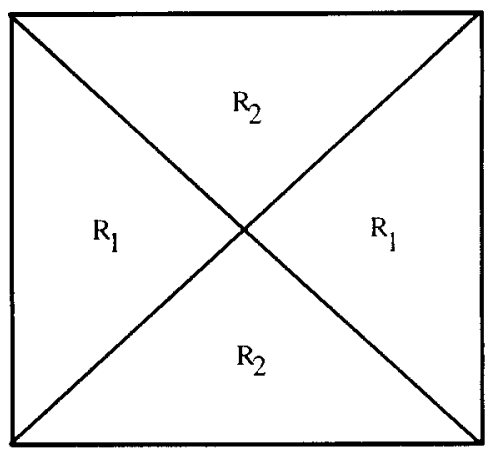

Fig. 3. Definition of $R_{1}$ and $R_{2}$.

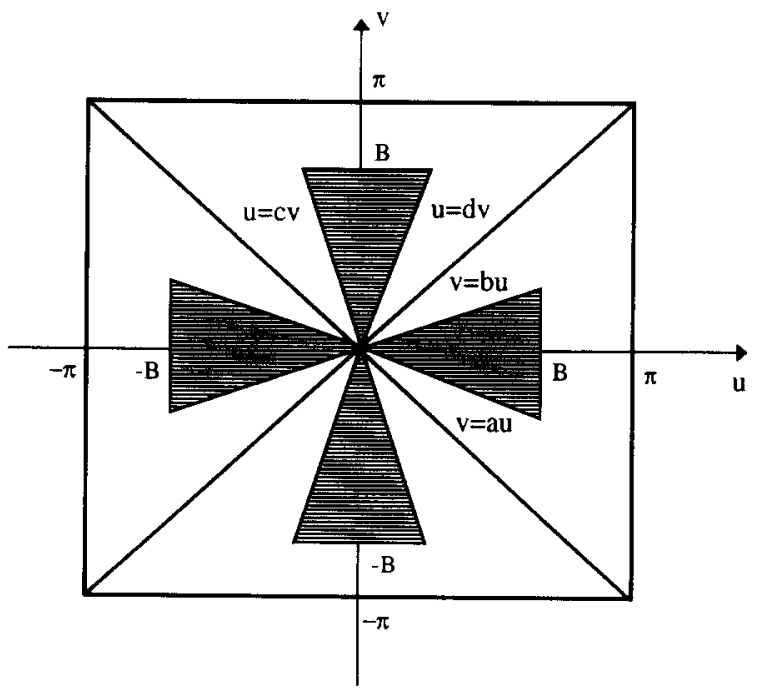

Fig. 4. Detail definition of passband in $R_{1}$ and $R_{2}$.

The two integrations in Eq. (3) can be replaced by only one integration with different arguments, so Eq. (3) can be written as

$h(m, n)=F(m, n, a, b, B)+F(m, n, c, d, B)$,

where the function $F$ is defined by

$F(m, n, p, q, B)=\frac{1}{4 \pi^{2}} \int_{0}^{B} \int_{p u}^{q u} 2 \cos (m u+n v) \mathrm{d} u \mathrm{~d} v$. 
The closed form of Eq. (5) for each special condition is

$$
\begin{aligned}
& F(m, n, p, q, B)=\frac{1}{4 \pi^{2}}\left\{\frac{2}{n m+n q}[1-\cos (m B+n q B)]\right. \\
& \left.-\frac{2}{n} \frac{1}{m+n p}[1-\cos (m B+n p B)]\right\} \\
& \text { for } n \neq 0, m+n q \neq 0, m+n p \neq 0 \text {, } \\
& F(0,0, p, q, B)=(q-p) B^{2} \frac{1}{4 \pi^{2}} \quad \text { for } m=n=0, \\
& =\frac{1}{4 \pi^{2}} \frac{2(q-p)}{m^{2}}[m B \sin (m B)+\cos (m B)-1]
\end{aligned}
$$

$F(m, n, p, q, B)=\frac{1}{4 \pi^{2}} \frac{2}{n} \frac{1}{m+n q}[1-\cos (m B+n p B)]$

for $m+n p=0$,

$F(m, n, p, q, B)$

$$
=\frac{1}{4 \pi^{2}} \frac{-2}{n} \frac{1}{m+n p}[1-\cos (m B+n p B)]
$$

for $m+n q=0$.

The ideal impulse response can now be windowed to produce a finite size filter, i.e.,

$g(m, n)=w(m, n) h(m, n)$,

where $w(m, n)$ is a $2 \mathrm{D}$ window function which is nonzero only in a finite area. The 2D window function generated by the McClellan transformation $[18,20]$ preserves the characteristics of the original 1D window function. Therefore, the 2D McClellan's transform Hamming window is used in this paper for general fan-type filter design.

\subsection{General fan-type filter design example}

One $25 \times 25$ size example is given to illustrate the efficiency and the power of this design method. A wide pass-angle $\left(\theta=110^{\circ}\right)$ fan-type filter is

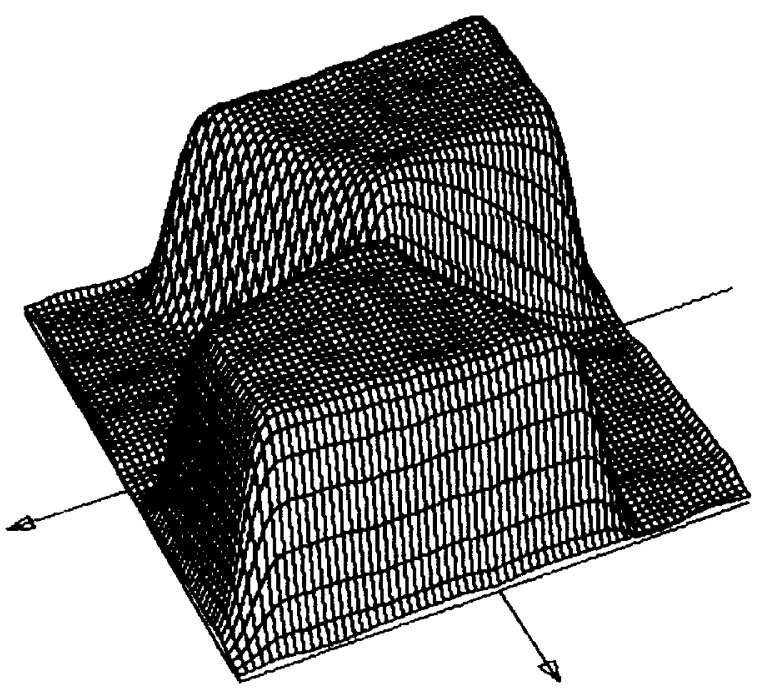

Fig. 5. Wide pass-angle general fan-type filter design by analytic method $\left(10^{\circ}-120^{\circ}, \theta=110^{\circ}, B=0.9 \pi\right)$.

designed in Fig. 5 to show the flexibility of this approach. The CPU design time of this example is $3.32 \mathrm{~s}$ on $\mathrm{VAX} / 780$ mini computer.

\subsection{Advantage and disadvantage}

(i) Advantages

(1) More flexible than the conventional transformed method: pass-direction, pass-angle and passband width are all variable to fit its individual requirements.

(2) Analytic closed form solution exists, very efficient in filter design.

(ii) Disadvantage

(1) The origin point is on the boundary between passband and stopband, so that it is attenuated after multiplying with a window function.

\section{Rotated fan-type filter}

\subsection{Introduction}

In order to overcome the above disadvantage of the attenuation at the zero-frequency response, we can locate the origin in the guard passband region, 
the zero frequency response is thus improved. A straightforward consideration is to define the passband region as in Fig. 6, then calculate its ideal impulse response. This will lead to very complicated expression and numerical difficulty. Instead a horizontal fan-type filter is defined as in Fig. 7. Its ideal impulse response can be easily calculated. Then by coordinate transformation, the calculated ideal impulse response can be rotated to any direction. This is called 'the rotated fan-type filter'.

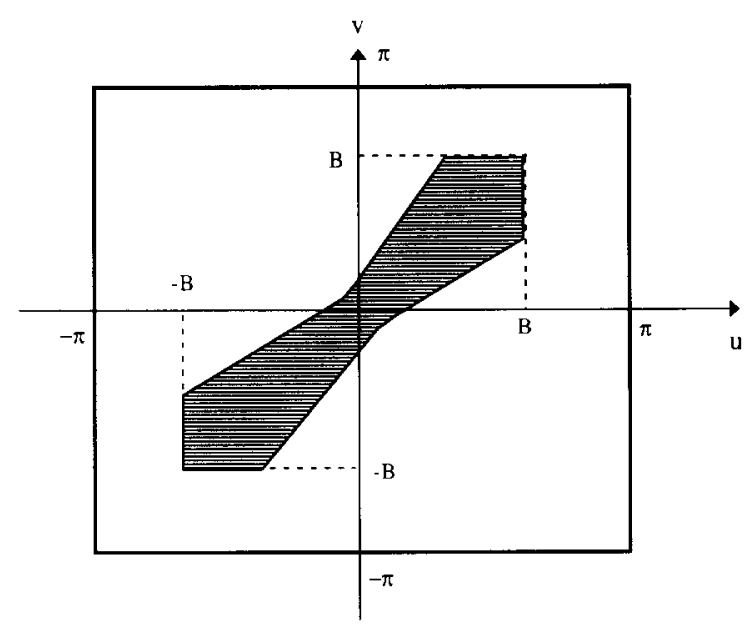

Fig. 6. Modified general fan-type filter with guard passband.

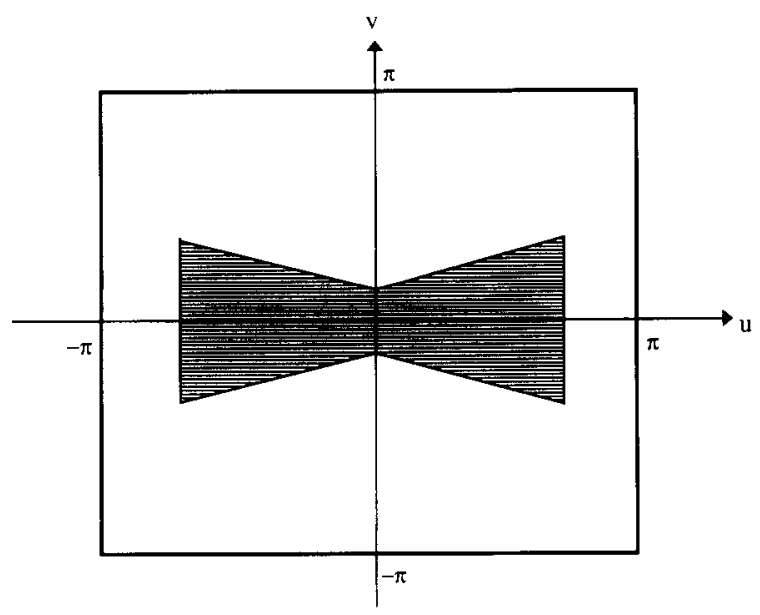

Fig. 7. Horizontal fan-type filter.

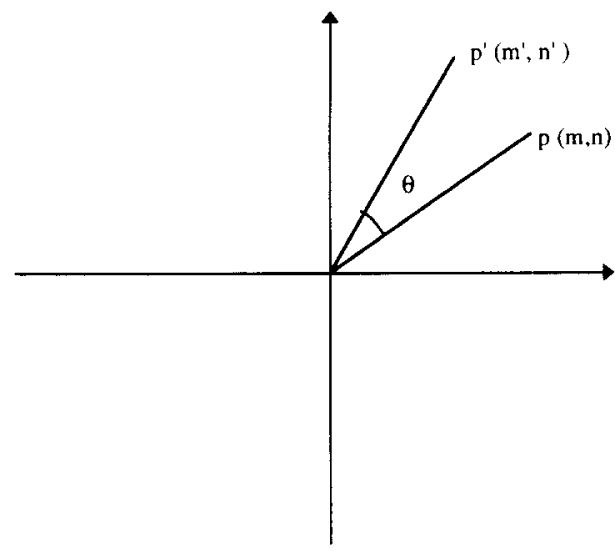

Fig. 8. Rotation operation.

\subsection{Rotation operation}

Rotation is a type of coordinate transformation. Suppose $(m, n)$ is the coordinate of point $p$, and $\left(m^{\prime}, n^{\prime}\right)$ is the coordinate of point $p^{\prime}$ which is rotated from point $p$ by an angle $\theta$ (see Fig. 8). Then the rotation can be written as

$\left(m^{\prime}, n^{\prime}\right)=(m, n)\left[\begin{array}{rr}\cos \theta & \sin \theta \\ -\sin \theta & \cos \theta\end{array}\right]$.

Note that $\left(m^{\prime}, n^{\prime}\right)$ may not be the integer point after this rotation. An integer point is defined as a point such that all of its coordinate components are integers.

\subsection{Ideal impulse response of rotated fan-type filter}

In the last section, rotation from an integer point may result in a non-integer point. Therefore interpolation may be needed to interpolate the value of the nearby integer points from the rotated results. But the interpolation operation is very complicated and time-consuming. Therefore a novel approach is developed to avoid this interpolation as below.

The detailed definition of a rotated fan-type filter is shown in Fig. 9(a). In order to overcome the attenuation at the zero-frequency response, a secondary-passband with value 1 is introduced to guard the main passband from the stopband. Two 

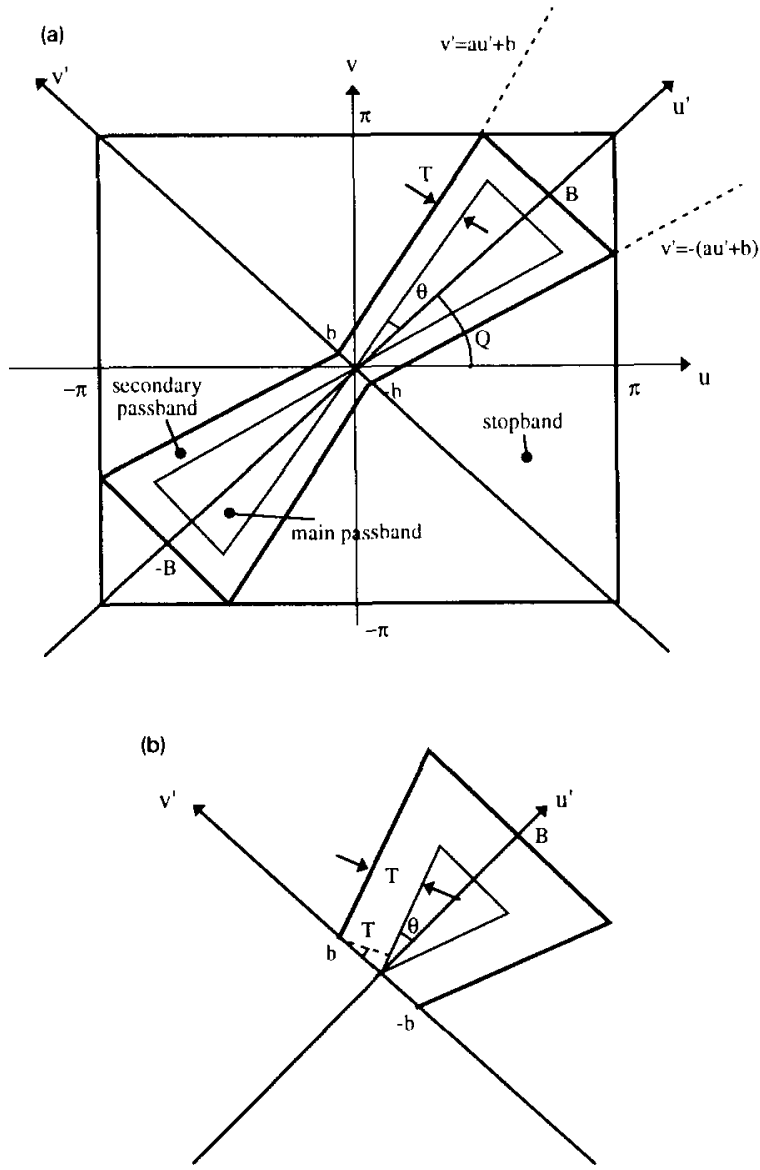

Fig. 9. (a) Detailed definition of a rotated fan-type filter. (b) Enlarged portion of Fig. 9(a).

parameters $a, b$ will be used later and are defined from Fig. 9(b) as

$a=\tan \theta$,

$b=\frac{T}{\cos \theta}$,

where $T$ is the width of the secondary passband. Then the ideal impulse response $h(m, n)$ can be written as

$h(m, n)=\frac{1}{4 \pi^{2}} \int_{-\pi}^{\pi} \int_{-\pi}^{\pi} H\left(\mathrm{e}^{\mathrm{j} u}, \mathrm{e}^{\mathrm{j} v}\right) \mathrm{e}^{\mathrm{j}(m u+n v)} \mathrm{d} u \mathrm{~d} v$.

In the original $(u, v)$ coordinate system, Eq. $(15)$ is difficult to integrate, but in the rotated $\left(u^{\prime}, v^{\prime}\right)$ co- ordinate system, the shape of the passband becomes a horizontal fan filter which is easier to integrate in the $\left(u^{\prime}, v^{\prime}\right)$ space. The relation between these two coordinate system is just a rotation operation and can be written as

$\left(u^{\prime}, v^{\prime}\right)=(u, v)\left[\begin{array}{rr}\cos Q & \sin Q \\ -\sin Q & \cos Q\end{array}\right]$,

where $Q$ is the angle between the two axes $u$ and $u^{\prime}$.

Taking $\left(u^{\prime}, v^{\prime}\right)$ into $(u, v)$, and using the Jacobian

$$
\begin{aligned}
\mathrm{d} u \mathrm{~d} v & =\left|\begin{array}{ll}
\frac{\partial u}{\partial u^{\prime}} & \frac{\partial v}{\partial u^{\prime}} \\
\frac{\partial u}{\partial v^{\prime}} & \frac{\partial v}{\partial v^{\prime}}
\end{array}\right| \mathrm{d} u^{\prime} \mathrm{d} v^{\prime} \\
& =\left|\begin{array}{rr}
\cos Q & \sin Q \\
-\sin Q & \cos Q
\end{array}\right| \mathrm{d} u^{\prime} \mathrm{d} v^{\prime} \\
& =\mathrm{d} u^{\prime} \mathrm{d} v^{\prime},
\end{aligned}
$$

then Eq. (15) can be rewritten as

$$
\begin{aligned}
h(m, n) & =\frac{2}{4 \pi^{2}} \int_{0}^{B} \int_{-\left(a u^{\prime}+b\right)}^{a u^{\prime}+b} \cos \left[m u^{\prime} \cos Q\right. \\
& \left.-m v^{\prime} \sin Q+n u^{\prime} \sin Q+n v^{\prime} \cos Q\right] \mathrm{d} v^{\prime} \mathrm{d} u^{\prime}, \\
& =\frac{2}{4 \pi^{2}} \int_{0}^{B} \int_{-\left(a u^{\prime}+b\right)}^{a u^{\prime}+b} \cos \left(p u^{\prime}+q v^{\prime}\right) \mathrm{d} v^{\prime} \mathrm{d} u^{\prime}, \\
& =g(p, q),
\end{aligned}
$$

where

$$
(p, q)=(m, n)\left[\begin{array}{rr}
\cos Q & -\sin Q \\
\sin Q & \cos Q
\end{array}\right] .
$$

Eq. (18) can be integrated and simplified into several closed form solutions according to various special conditions.

$$
\begin{aligned}
& g(p, q)=\frac{1}{4 \pi^{2} q}\left[\frac{\cos (q b)-\cos (p B+q a B+q b)}{p+q a}\right. \\
& \left.-\frac{\cos (q b)-\cos (p B-q a B-q b)}{p-q a}\right]
\end{aligned}
$$

for $q \neq 0, p+q a \neq 0, p-q a \neq 0$,

$g(0,0)=\frac{B}{4 \pi^{2}}(a B+2 b)$ for $p=q=0$, 


$$
g(p, 0)=\frac{1}{2 \pi^{2} p}\left[(a B+b) \sin (p B)+\frac{a(\cos (p B)-1)}{p}\right]
$$$$
\text { for } q=0 \text {, }
$$

$$
\begin{aligned}
g(p, q)=\frac{1}{4 \pi^{2} q}[ & B \sin (q b) \\
& \left.-\frac{\cos (q b)-\cos (p B-q a B-q b)}{p-q a}\right]
\end{aligned}
$$

for $p+q a=0$,

$$
\begin{aligned}
g(p, q)=\frac{1}{4 \pi^{2} q}[ & B \sin (q b) \\
& \left.+\frac{\cos (q b)-\cos (p B+q a B+q b)}{p+q a}\right]
\end{aligned}
$$

for $p-q a=0$.

\subsection{Rotated fan-type filter design example}

Since the rotation method cannot design quadrant fan-type filters, only one design example is provided. Fig. 10 shows the designed narrow passangle $\left(\theta=30^{\circ}\right)$ fan-type filter's frequency response.

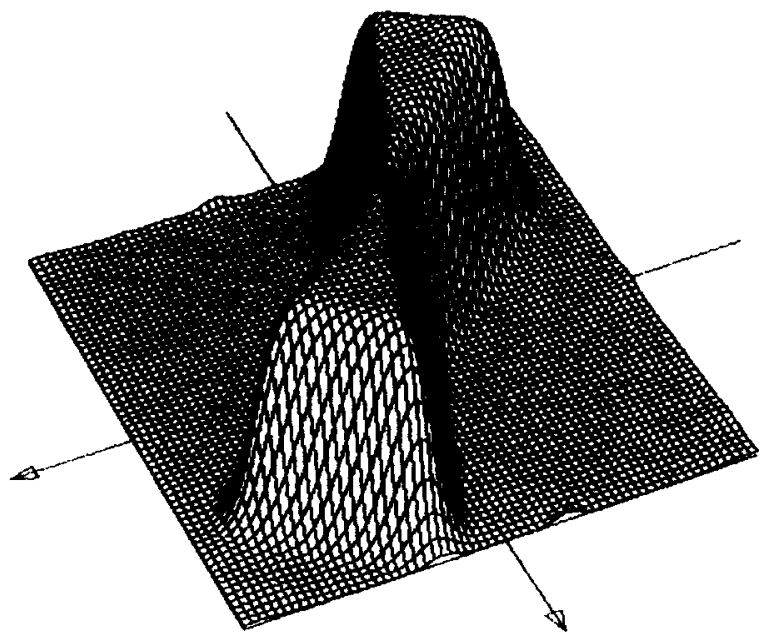

Fig. 10. Narrow pass-angle fan-type filter design by rotated method $\left(\theta=30^{\circ}, Q=55^{\circ}, B=0.8 \pi, T=0.1 \pi\right)$.
Note that the zero-frequency response has been much improved relative to the previous cases. The CPU time of this example is very fast with $3.23 \mathrm{~s}$ only.

\subsection{Advantage and disadvantage}

(i) Advantage

(1) Since a secondary passband is inserted between the main passband and the stopband, the zero-frequency response has been improved.

(2) Since there exists a closed form, this is an efficient design method.

(ii) Disadvantage

(1) The passband region cannot extend to the corner, so some high-frequency information is lost.

(2) There is no general rule to specify the width of the secondary passband, it may be needed to try several times to get a proper design.

\section{Iterated frequency sampling fan type filter}

\subsection{Iterative design algorithm}

Frequency sampling approach is a straightforward method to design digital filters. It utilizes the inverse fast Fourier transform (IFFT) to perform the main task. First the frequency response $H(k, l)$ is assigned with an ideal value for each frequency sampling point $(k, l)$. If the point $(k, l)$ locates on passband region, $H(k, l)$ is assigned with 1 , otherwise it is assigned with 0 , then $H(k, l)$ is taken the 2D IFFT procedure to obtain $h(m, n)$, this filter impulse response is not optimal, we propose a modified frequency sampling method that iteratively modifies $h(m, n)$ according to the difference between the actual and the ideal frequency responses. This method is based on the successive projections technique $[1,3,19]$ by using both timeand frequency-domain constraints. This technique is very simple and can easily be implemented using the FFT algorithm. 
The detailed steps to find $h(m, n)$ by the iterative frequency sampling method are the following. Step 1. Initializations.

(a) specify desired ideal frequency response DESIRE $(k, l)$.

specify window function $w(m, n)$.

specify maximum iteration times MAXITE.

set RIPPLE to be infinite.

set iterated times ITERAT to be 0 .

(b) test $(m, n) \leftarrow$ (IFFT) $\leftarrow$ DESIRE $(k, l)$.

(c) test $(m, n) \leftarrow \operatorname{test}(m, n) w(m, n)$

Step 2. Testing the performance.

(a) test $(m, n) \rightarrow($ FFT $) \rightarrow$ TEST $(k, l)$.

(b) if $(k, l)$ is in transition band then DIFF $(k, l) \leftarrow 0$, else DIFF $(k, l) \leftarrow$ DESIRE $(k, l)-$ TEST $(k, l)$, end if.

(c) find the maximum value MAXERR of $|\operatorname{DIFF}(k, l)|$.

(d) if MAXERR > RIPPLE then go to Step 5.

(e) $h(m, n) \leftarrow$ test $(m, n)$.

(f) RIPPLE $\leftarrow$ MAXERR.

Step 3. Iteration limit check.

(a) ITERAT $\leftarrow$ ITERAT +1 .

(b) if ITERAT = MAXITE then go to Step 5.

Step 4. Impulse response's differential correction.

(a) $\operatorname{DIFF}(k, l) \rightarrow($ IFFT $) \rightarrow \operatorname{diff}(m, n)$.

(b) test $(m, n) \leftarrow$ test $(m, n)+\operatorname{diff}(m, n) \cdot w(m, n)$.

(c) go to Step 2.

Step 5. Termination

$h(m, n)$ is the designed filter's impulse response.

Finally, we would discuss the convergence of this design algorithm, from our experiments we found that MAXERR becomes smaller and smaller until reaching the smallest value and then begins to increase, so the design procedure is terminated after MAXERR reaching the smallest value (see Step 2(d) in the above).

\subsection{Modified specification of general fan-type filter}

The definition of general fan-type filter is modified slightly in order to improve the zero-frequency response. The frequency domain is divided into passband, stopband and transition band (see Fig. 11), respectively.

When using frequency sampling, the initial value of transition band may effect the converging speed.

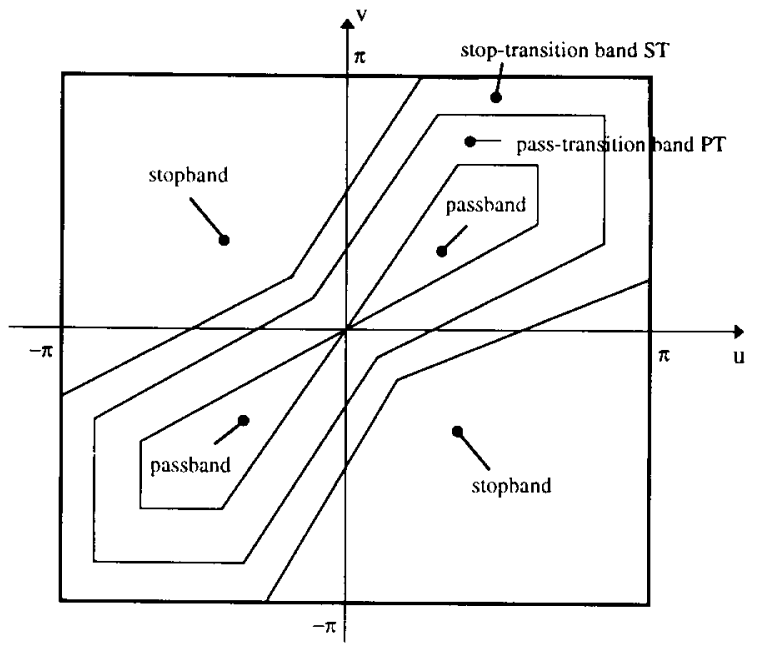

Fig. 11. Specification of general fan-type filter by frequency sampling method.

Our method is to divide the transition band into pass-transition band and stop-transition band, pass-transition band is assigned with 1 , and stoptransition band is assigned with 0 , these two transition bands are used to protect the passband and stopband, and prevent the leakage from the adjacent bands.

\subsection{Frequency sampling fan type filter design example}

An example designed from this approach is shown in Fig. 12. The CPU design time of this example is about $35.1 \mathrm{~s}$ in 8 iterations, and is longer than the previous two closed form approaches.

\subsection{Advantage and disadvantage}

(i) Advantage

(1) Frequency sampling method can design any arbitrary shape 2D FIR filters.

(2) Proposed frequency sampling method can iteratively modify the filter $h(m, n)$ to be a better approximation of ideal frequency response. 


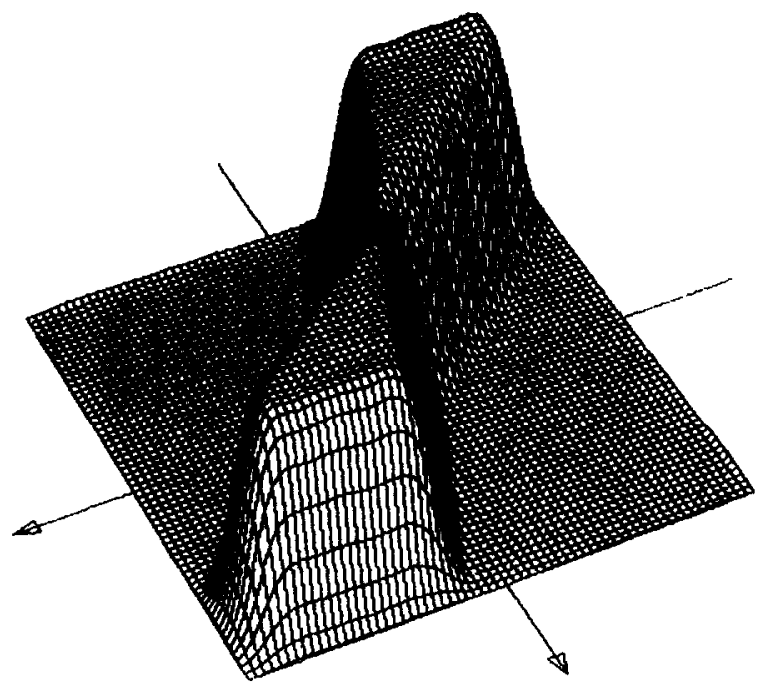

Fig. 12. Narrow pass-angle fan-type filter design by frequency sampling method $\left(40^{\circ}-70^{\circ}, \theta=30^{\circ}, P T=S T=0.1 \pi\right)$.

\section{(ii) Disadvantage}

(1) Iterated frequency sampling method consumes much more time than the analytic and rotated methods.

(2) The rule to specify transition bandwidth is not unique, it may be needed to try several times to get a proper design.

\section{Conclusions}

In this paper, we have described three efficient approaches for designing general arbitrary passangle fan-type filters, these include the analytic closed form, rotated and frequency sampling fantype FIR filters, each filter has its own unique features and advantages suitable for some applications.

The major application of fan-type filters is directional filtering, which will select the signals in prescribed directions to pass and reject the other ones. In addition fan-type filters [8] are also useful for second generation high compression image coding $[11,12]$, it can detect the edge informations in all given directions very successfully.

\section{References}

[1] A. Abo-Taleb and M.M. Fahmy, "Design of FIR twodimensional digital filters by successive projections", IEEE Trans. Circuits and Systems, Vol. CAS-31, September 1984, pp. 801-805.

[2] R. Ansari, "Efficient IIR and FIR fan filters", IEEE Trans. Circuits and Systems, Vol. CAS-34, August 1987, pp.941-945.

[3] A.E. Cetin and R. Ansari, "Iterative procedure for designing two-dimensional FIR filters", Electron. Lett., Vol. 23, No. 3, January 1987, pp. 131-133.

[4] H.P. Chang and J.K. Aggarwal, "Design of two-dimensional recursive filters by interpolation", IEEE Trans. Circuits and Systems, Vol. CAS-24, June 1977, pp. 281-291.

[5] M.P. Ekstrom, R.E. Twogood and J.W. Woods, "Two-dimensional recursive filter design - a spectral factorization approach", IEEE Trans. Acoust. Speech Signal Proces., Vol. ASSP-28, February 1980, pp. 16-26.

[6] J.P. Fail and G. Grau, "Les filters en eventail", Geophys. Prospecting, Vol. 11, 1963, pp. 131-163.

[7] A. Fettweis, "Design of recursive quadrant filters", Archiv Elektr. Ubertr., Vol. 34, March 1980, pp. 97-103.

[8] A. Fettweis, T. Leickel, M. Bolle and U. Sauvagerd, "Realization of filter banks by means of wave digital filters", Proc. IEEE Internat. Symp. on Circuits and Systems, New Orleans, LA, USA, May 1990, pp. 2013-2016.

[9] A.P. Gerheim, 'Synthesis procedure for $90^{\circ}$ fan filters", IEEE Trans. Circuits and Systems, Vol. CAS-30, December 1983, pp. 858-864.

[10] A.H. Kayran and R.A. King, "Design of recursive and nonrecursive fan filters with complex transformations", IEEE Trans. Circuits and Systems, Vol. CAS-30, December 1983, pp. 849859.

[11] M. Kunt, M. Benard and R. Leonardi, "Recent results in high-compression image coding", IEEE Trans. Circuits and Systems, Vol. CAS-34, November 1987, pp. 1306-1336.

[12] M. Kunt, A. Ikonomopoulos and M. Kocher, "Second generation image codin'g techniques", Proc. IEEE, Vol. 73, April 1985, pp. 549-574.

[13] G. Linnenberg, "Wave digital fan filters: Numerical and experimental results", in: M. Kunt and F. De Coulon, eds., Signal Processing: Theories and Applications, EURASIP, North-Holland, Amsterdam, 1980, pp. 15-19.

[14] J.H. Lodge and M.M. Fahmy, "An efficient $L_{p}$ optimization technique for the design two-dimensional linear phase FIR digital filters", IEEE Trans. Acoust. Speech Signal Process., Vol. ASSP-28, June 1980, pp. 308-313.

[15] J.H. McClellan, "The design of 2D digital filters by transformations", Proc. 7th Annual Princeton Conf. Inform. Sci. and Systems, 1973, pp. 247-251.

[16] R.M. Mersereau, "The design of arbitrary 2D zero-phase FIR filters using transformations", IEEE Trans. Circuits and Systems, Vol. CAS-27, February 1980, pp. 142-144.

[17] M.Z. Mulk, K. Hirano and K. Obata, "Design of digital fan filters", IEEE Trans. Acoust. Speech Signal Process., Vol. ASSP-31, December 1983, pp. 1427-1435. 
[18] S.C. Pei and S.B. Jaw, "A novel 2-D window for spectral estimation", IEEE Trans. Circuits and Systems, Vol. CAS34, September 1987, pp. 1112-1115.

[19] S.C. Pei and I.I. Yang, "Design of a class of time-constrained FIR digital filters by successive projections",
IEEE Trans. Circuits and Systems, Vol. CAS-36, January 1989, pp. 164-167.

[20] T.H. Yu and S.K. Mitra, "A new two-dimensional window”, IEEE Trans. Acoust. Speech Signal Process., Vol. ASSP-33, August 1985, pp. 1058-1061. 